\title{
Colangiocarcinoma hiliar (tumor de Klatskin)
}

\author{
Fernando Hidalgo Méndez.
}

Servicio de Diagnóstico por la Imagen. CAP El Serral (Instituto Catalán de la Salud).

Sant Vicenç dels Horts.

Barcelona (España).

Correspondencia:

Fernando Hidalgo Méndez.

Servicio de Diagnóstico por la

Imagen. CAP El Serral.

Ctra. Sant Boi, 59-61.

C.P. 08620 Sant Vicenç dels

Horts. Barcelona (España).

Correo electrónico:

26415fhm@comb.cat

Recibido el 26 de diciembre de 2013.

Aceptado para su publicación el 27 de enero de 2014.

\begin{abstract}
RESUMEN
El colangiocarcinoma es una neoplasia maligna del epitelio de la vía biliar. Su frecuencia de aparición es baja, y su pronóstico es infausto en un breve periodo de tiempo (meses) en la mayoría de los pacientes afectos. La poca especificidad de los síntomas clínicos en su presentación y la infiltración tumoral local en el momento del diagnóstico son factores que suelen impedir la posibilidad de ofrecer una resección quirúrgica radical que sea potencialmente curativa.

El caso de una paciente de 52 años afecta de un colangiocarcinoma de la confluencia hiliar (tumor de Klatskin) nos sirve para revisar los aspectos epidemiológicos y clínicos de esta entidad, así como los datos de laboratorio, hallazgos en pruebas de imagen, diagnóstico diferencial y tratamiento potencial de esta infrecuente enfermedad.
\end{abstract}

Palabras clave: Conductos Biliares. Colangiocarcinoma. Tumor de Klatskin.

\section{ABSTRACT}

Hilar cholangiocarcinoma (Klatskin tumor)

Cholangiocarcinoma is a malignant neoplasm of the hepatic bile duct epithelium. The frequency of occurrence is low and has very poor prognosis (short-term survival in months) in most patients affected. The lack of specificity in the clinical symptoms at onset and local tumor infiltration at the time of diagnosis are factors which usually prevent the possibility to offer radical surgical resection as potentially curative.

The case of a 52 years old patient suffering from cholangiocarcinoma in the hilar confluence (Klatskin tumor) serves as the basis to review epidemiological and clinical aspects of this entity, as well as laboratory information, findings of imaging tests, differential diagnosis and potential treatment for this uncommon disease.

Keywords: Bile Ducts. Cholangiocarcinoma. Klatskin's tumor.

\section{INTRODUCCIÓN}

El carcinoma de vías biliares (colangiocarcinoma) es una entidad infrecuente que representa menos del $2 \%$ de todos los tumores malignos ${ }^{1-3}$, con una incidencia de entre 1 y 2 casos nuevos por cada 100.000 habitantes y año $0^{1,2}$. La edad de presentación más frecuente es a partir de la sexta década de la vida $^{1-3}$, siendo ligeramente más habitual en hombres que en mujeres $(1,3 / 1)^{1,4}$. El tipo histológico más habitual es el adenocarcinoma ductal bien diferenciado secretor de mucina ${ }^{1,4}$.

El colangiocarcinoma puede aparecer en cualquier punto del árbol biliar (desde los canalículos intrahepáticos hasta la ampolla de Vater), siendo el tramo perihiliar (zona de la confluencia de los conductos hepáticos principales) el más frecuentemente afectado, representando entre un 40 y un $60 \%$ del total ${ }^{1-5}$; a este subtipo se le denomina tumor de Klatskin (colangiocarcinoma hiliar). 
A día de hoy se trata aún de una entidad de pronóstico habitualmente infausto y en la que la única terapia potencialmente curativa (la resección radical) sólo puede ser aplicada en una minoría de pacientes ${ }^{1,3,5}$. No obstante, los avances recientes en el diagnóstico por imagen y en las técnicas quirúrgicas aplicadas han aumentado significativamente la posibilidad de una esperanza de curación cada vez mayor ${ }^{6}$. Así pues, es el propósito de este artículo servir de revisión (tanto a médicos de asistencia primaria como a especialistas en el diagnóstico por la imagen) de los rasgos fundamentales de esta infrecuente entidad para que una actuación conjunta nos permita ofrecer progresivamente un mejor pronóstico a estos pacientes.

\section{OBSERVACIONES CLÍNICAS}

Paciente mujer de 52 años, hipertensa en tratamiento farmacológico, sin hábitos tóxicos ni otros antecedentes significativos. Acude a nuestro centro de Atención Primaria presentando un cuadro de 15 días de evolución de dolor en el hipocondrio derecho y prurito de intensidad progresiva, acompañado de sensación febricular, astenia, pérdida del apetito, coluria y acolia. En la exploración física se objetiva un tinte ictérico conjuntival y muco-cutáneo. La analítica muestra hiperbilirrubinemia (93 $\mathrm{mmol} / \mathrm{L}$ ), hipertransaminemia (AST: $114 \mathrm{U} / \mathrm{L} ; \mathrm{ALT}$ : $263 \mathrm{U} / \mathrm{L}$ ) y elevación de la fosfatasa alcalina (808 $\mathrm{U} / \mathrm{L})$.
En la ecografía abdominal se aprecia la existencia de una masa sólida hepática, hipoecogénica, de 64 milímetros de diámetro mayor, con dilatación de la vía biliar intrahepática izquierda (figura 1). En la tomografia computerizada (TC) dicha imagen aparece como una lesión tumoral sólida, centrada en el segmento IV (con extensión a los segmentos III y V), predominantemente hipodensa, con un realce periférico e irregular de contraste en las diferentes fases, englobando la rama porta izquierda e infiltrando las ramas arteriales izquierdas, con dilatación retrógrada de los conductos biliares izquierdos y, levemente, de las vías biliares del sector posterior derecho; no se aprecian signos de extensión ganglionar locorregional ni de diseminación a distancia (figura 2).

Una biopsia hepática guiada por ecografía obtiene un material compatible con adenocarcinoma.

Se indica un drenaje biliar mediante una colangiografía transparietohepática, consiguiendo colocar sólo un drenaje interno-externo a nivel del conducto hepático anterior derecho, no siendo posible drenar otros conductos por falta de dilatación o por infiltración tumoral. Tras un episodio de colangitis aguda con hemocultivos positivos para Pseudomonas aeruginosa (tratado con amikacina y ceftazidima, según antibiograma), la paciente presenta buena evolución con mejoría de la ictericia, disminución progresiva de la hiperbilirrubinemia, ausencia de fiebre y disminución del débito del drenaje biliar.

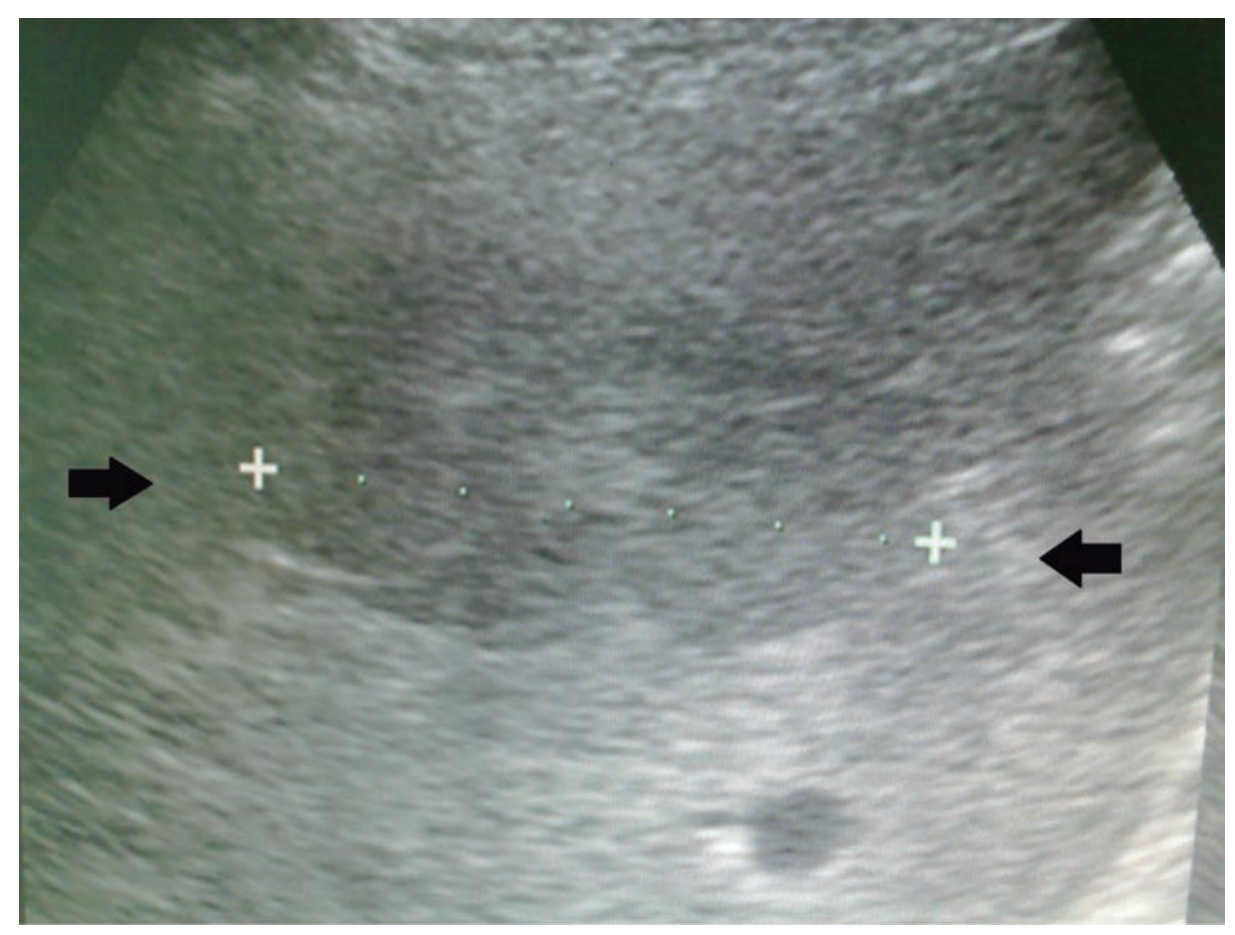

Figura 1. Ecografía abdominal. Masa hepática (entre flechas) sólida, hipoecogénica, de $64 \mathrm{~mm}$. de diámetro mayor. 
Se realiza un tratamiento quirúrgico radical (con intención curativa) practicando una hepatectomía izquierda ampliada con resección de la vía biliar y reconstrucción del tránsito biliar mediante una hepatoyeyunostomía. El diagnóstico histológico postoperatorio es de colangiocarcinoma hiliar o tumor de Klatskin (grupo Illb en la clasificación de Bismuth-Corlette).

En los meses siguientes a la intervención quirúrgica la paciente presenta cuadros recurrentes de ascitis (que requieren ingresos hospitalarios) y desarrollo de encefalopatía, falleciendo a los trece meses del inicio de la sintomatología y a los diez meses de la cirugía.

\section{COMENTARIOS}

El colangiocarcinoma es un proceso maligno infrecuente originado en el epitelio de la vía biliar, cuya historia natural lleva a la mayoría de los pacientes a fallecer entre seis y doce meses después del inicio de los síntomas ${ }^{3}$. La resección quirúrgica radical de la lesión (único tratamiento potencialmente curativo) no es posible en la mayoría de los casos dado el grado de infiltración local en el momento del diagnóstico y los efectos de la obstrucción biliar tumoral (colangitis, ulterior insuficiencia hepática y renal) ${ }^{1,3,5}$. La irresecabilidad quirúrgica es más frecuente en los tumores que afectan la confluencia biliar hiliar que en los que se sitúan en tramos más distales de la vía biliar, empeorando aún más el pronóstico ${ }^{3}$.

Como en el caso de nuestra paciente, la clínica de presentación es inespecífica ${ }^{1-5}$. Los pacientes pueden referir dolor en el hipocondrio derecho, pérdida de peso, anorexia, prurito, acolia, coluria e ictericia; esta última no aparecerá inicialmente si la obstrucción biliar no es completa o afecta sólo a conductos segmentarios ${ }^{2}$. Los datos de laboratorio muestran un patrón de ictericia obstructiva, con hiperbilirrubinemia y con aumento de los valores de la fosfatasa alcalina, la gamma-glutamil-transpeptidasa y las transaminasas ${ }^{1,3-5}$. En los casos más avanzados puede haber una disminución del hematocrito y anomalías en las pruebas de coagulación ${ }^{1}$. No existe ningún marcador tumoral específico para el colangiocarcinoma, ya que sus valores pueden incrementarse también en otras entidades malignas (neoplasias hepatocelulares, gástricas, colorrectales y esofágicas) o benignas (pancreatitis, colangitis agudas); no obstante, el CA 19-9 ha sido descrito como el marcador que aumenta más sus niveles en caso de carcinoma de la vía biliar ${ }^{1,4,5}$.

En el diagnóstico diferencial del colangiocarcinoma se incluyen entidades como el carcinoma de la vesícula biliar, el síndrome de Mirizzi (impactación de una litiasis en el cuello vesicular), las estenosis focales benignas de la vía biliar ("malignant masquerade") y las metástasis biliares de otras neoplasias (páncreas, estómago, mama, pulmón o colon) ${ }^{1,2}$.

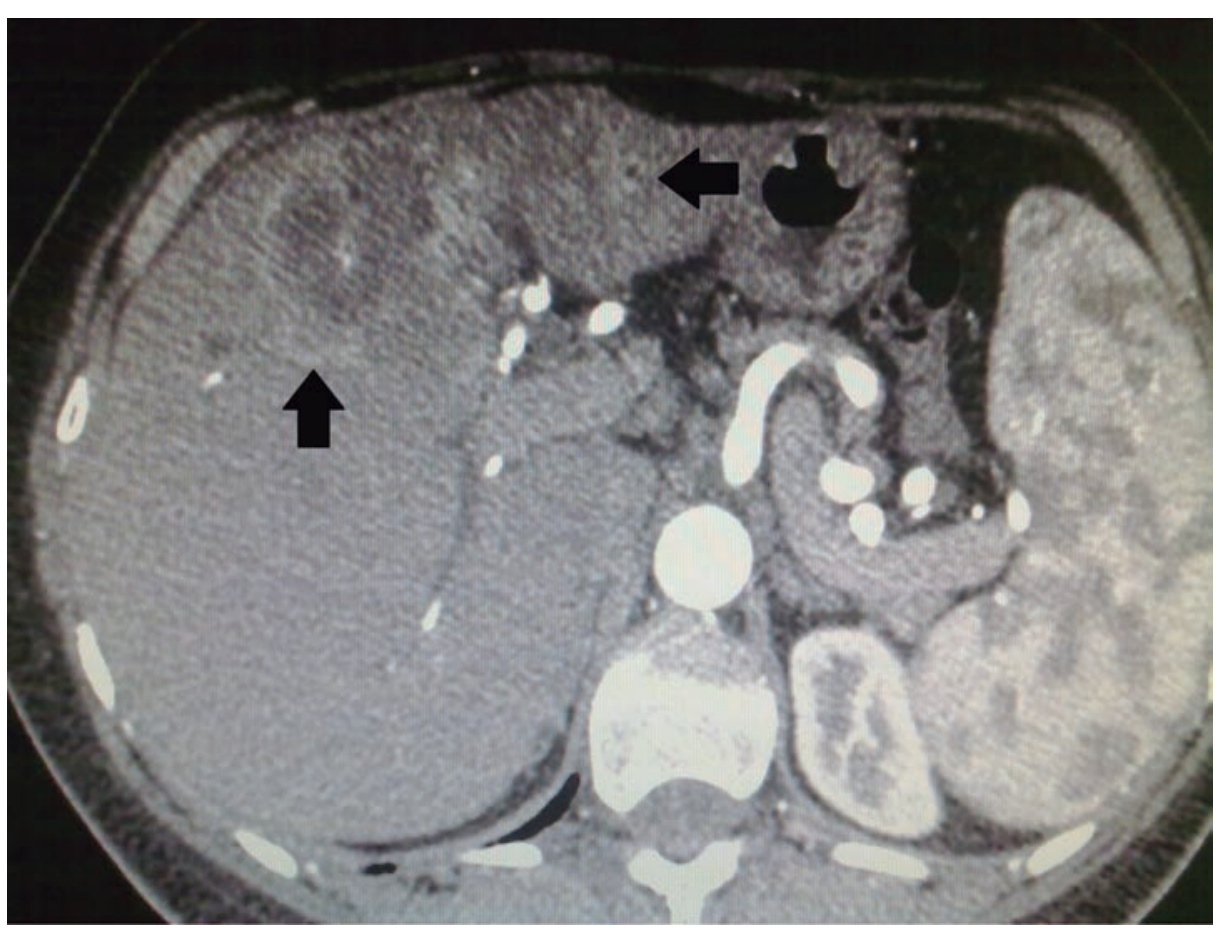

Figura 2. Tomografía computerizada de abdomen con contraste. Masa tumoral (entre flechas) sólida, predominantemente hipodensa, con realce periférico irregular al administrar el contraste, centrada en el segmento IV (con extensión a los segmentos III y V), en contacto con la convergencia biliar principal. 
La etiología del colangiocarcinoma es desconocida y la mayoría de los casos son presentaciones esporádicas. No obstante, se han descrito una serie de entidades que supondrían un mayor riesgo potencial de desarrollar esta neoplasia ${ }^{1-5}$, entre ellas destaca la colangitis esclerosante primaria, con una asociación con el colangiocarcinoma de entre un $0,6 \%$ y un $1,5 \%$ anual y con una presentación de la neoplasia a edades más tempranas de lo habitual ${ }^{5}$. Otras entidades potencialmente de riesgo son la enfermedad quística biliar congénita, la litiasis intrahepática, algunos parásitos biliares en países de Oriente (Clonorchis sinensis y Ophisthorchis viverrini), la exposición química a tóxicos (thorotrast, digoxinas, nitrosaminas, asbesto), el síndrome de Lynch (cáncer colorrectal hereditario no asociado a pólipos) y la enfermedad vírica crónica hepática (con menor asociación con la neoplasia de la vía biliar que con el carcinoma hepatocelular). Es dudoso que exista asociación entre las colelitiasis (con o sin colecistectomía subsiguiente) y el desarrollo del colangiocarcinoma ${ }^{1}$.

En nuestra paciente la clínica de presentación (dolor en hipocondrio derecho, prurito, febrícula, astenia, anorexia, coluria y acolia) sugería una obstrucción inespecífica de la vía biliar. Ante la ausencia de factores predisponentes que inicialmente hiciesen sospechar una patología tumoral biliar, fue imprescindible la realización de técnicas de imagen y de un estudio histológico para concretar el diagnóstico.

El único tratamiento potencialmente curativo del colangiocarcinoma es la resección quirúrgica completa del tumor con márgenes histológicamente libres de enfermedad ${ }^{1-4}$. Aunque, en última instancia, será el acto quirúrgico el que determine la resecabilidad o la irresecabilidad de la lesión, se considera la existencia de cuatro criterios prequirúrgicos de potencial resecabilidad 5 : la no afectación de los ganglios retroperitoneales ni de la arteria celíaca; la no invasión de la vena porta ni de la arteria hepática principal; la ausencia de afectación de órganos vecinos; y la no diseminación a distancia de la enfermedad. Una TC abdominal constató que nuestra paciente cumplía con estos criterios (se apreció afectación de ramas portales y arteriales izquierdas, pero no de la vena porta ni de la arteria hepática principal), por lo que se planteó y realizó una actuación quirúrgica radical con intención curativa.

En el caso del colangiocarcinoma hiliar la técnica quirúrgica de elección es la resección de la vía biliar afectada, hepatectomías parciales (el lóbulo caudado suele estar invadido-5), linfadenectomía y anastomosis biliodigestiva ${ }^{1-6}$. Aunque el tratamiento quirúrgico del colangiocarcinoma hiliar ha mejorado significativamente en los últimos años gracias a las técnicas de diagnóstico precoz por imagen y a un mejor manejo perioperatorio de estos pacientes ${ }^{1,6}$, la resección sólo es posible en menos de la tercera parte de los casos $^{4}$. El tumor de Klatskin suele invadir localmente, con metástasis a distancia entre el $10 \%$ y el $30 \%$ de los casos $^{3}$. En caso de recidiva, ésta se sitúa en el hilio hepático, seguido por el hígado, pulmón, huesos y piel ${ }^{3}$. El tratamiento paliativo de elección en el tumor de Klatskin es la resolución de la ictericia y del prurito mediante un drenaje biliar por vía endoscópica como primera opción, o por vía percutánea como alternativa ante complicaciones del método endoscópico (mala colocación de las prótesis endoscópicas, colangitis $)^{3-5}$. La radioterapia, la quimioterapia y el transplante hepático no han mostrado una utilidad significativa de cara a mejorar la expectativa y la calidad de vida en los pacientes afectos de esta neoplasia ${ }^{1-5}$.

Dado que la presentación clínica del tumor de la vía biliar acostumbra a ser inespecífica y progresiva, es habitual que estos pacientes acudan inicialmente al Centro de Atención Primaria y sea el médico de familia quien oriente el proceso diagnóstico. Por ello, sirva este artículo como revisión de las características principales de esta entidad. Su conocimiento hará que, a pesar de dicha inespecificidad clínica, aumente la alerta ante la posibilidad de encontrarnos ante esta infrecuente neoplasia y pueda llegarse a un diagnóstico lo más precoz posible que aumente las opciones de ofrecer al paciente un tratamiento potencialmente curativo.

\section{BIBLIOGRAFÍA}

1. Burgos L. Colangiocarcinoma. Actualización, diagnóstico y terapia. Rev Med Chile. 2008; 136: 240-8.

2. Saldinger PF, Jarnagin WR, Blumgart LH. Hilar cholangiocarcinoma. En: Blumgart LH, editor. American Cancer Society Atlas of Clinical Oncology: Hepatobiliary Cancer. Shelton, CT: PMPH-USA Ltd.; 2001. p. 193-209.

3. Giménez M, Andreacchio A. Carcinoma de vías biliares. Tumor de Klatskin. En: Galindo F, editor. Cirugía digestiva. Buenos Aires: SACD; 2009. IV-466-, p. 1-12.

4. Brenes PC. Tumor de Klatskin. Rev Med Cos Cen. 2010; 592: 169-71.

5. Castaño-Llano R. Hilar Cholangiocarcinoma (Klatskin tumor). Rev Col Gastroenterol. 2011; 26 (2): 116-24.

6. Lu BC, Ren PT. Treatment of hilar cholangiocarcinoma of Bismuth-Corlette type III with hepaticojejunostomy. Wspolczesna Onkol. 2013; 17 (3): 298-301. 